\title{
Diversidad irresistible. Megan Raby estudia las estaciones biológicas estadounidenses en el Caribe en su libro American Tropics
}

\author{
Claudia Leal ${ }^{1}$
}

Book Review:

Megan Raby. American Tropics: The Caribbean Roots of Biodiversity Science.

(Chapel Hill: The University of North Carolina Press, 2017).

La historia ambiental latinoamericana surgió motivada por el convencimiento de que la naturaleza de la región es muy valiosa y se está perdiendo. Libros pioneros como los de Warren Dean-With Broadax and Firebrand-y Reinaldo Funes-De bosque a sabana-documentan la desaparición de ricos bosques tropicales. ${ }^{2}$ La noción que más ha avanzado esta idea de tesoro amenazado ha sido sin duda la de biodiversidad. Dicha noción no surgió por generación espontánea en la década de 1980, sino que tiene una historia fascinante que Megan Raby recuenta en este libro cautivador.

\footnotetext{
${ }^{1}$ Ph.D em Geografía, University of California - Berkeley, Estados Unidos. Profesora associada del Departamento de Historia de la Universidad de los Andes, Colombia. claleal@uniandes.edu.co

${ }^{2}$ Warren Dean, With Broadax and Firebrand: The Destruction of Brazil's Atlantic Forest (Berkeley: The University of North California Press, 1995); Reinaldo Funes Monzote, De bosque a sabana: azúcar, deforestación y medio ambiente en Cuba, 1942-1926 (México: Siglo Veintinuo Editores, Estado Libre y Soberano de Quintana Roo, 2000), publicado en inglés como From Rainforest to Cane Field in Cuba: An Environmental History since 1492 (Chapel Hill: The University of North Carolina Press, 2008).
} 
American Tropics es de lectura ágil, tiene trama y personajes $\mathrm{y}-\mathrm{al}$ igual que $\mathrm{A}$ Biologia Militante de Regina Horta Duarte-está en ese fértil cruce de caminos entre la historia de la ciencia y la historia ambiental. ${ }^{3}$ Recuenta cómo la diversidad del trópico llegó a ocupar un lugar central en los estudios ecológicos hechos en Estados Unidos bajo el rótulo de "ecología tropical". Para ello, desde los albores del siglo XX, biólogos de dicho país se encaminaron hacia el sur-sobre todo a lugares en el Gran Caribe-en busca de la naturaleza que pretendían entender, encarnada siempre por bosques. Allí establecieron unas pocas estaciones biológicas que hicieron posible su trabajo.

Estas estaciones fueron-y siguen siendo-la institución base de una forma de hacer ciencia que tomó fuerza en Europa desde finales del siglo XIX y que aboga por estudiar los organismos en sus ambientes naturales para así entender las relaciones entre ellos. Las estaciones permiten "el uso de técnicas experimentales [...], estudios de comportamiento e historias de vida, la realización repetida de censos de población y de inventarios taxonómicos detallados" (207). Además, hacen posible estudios de larga duración y se constituyen una empresa mancomunada de construcción de conocimiento sobre un mismo espacio hecho a muchas manos. (Quien estudia primates, por ejemplo, puede apoyarse en los resultados de quienes han estudiado las plantas del lugar). Inicialmente botánicos, pero pronto zoólogos también, comenzaron a argumentar que no podía haber una verdadera ciencia de la naturaleza sin estudiar sus manifestaciones más complejas que estaban, sin duda, en la diversidad de los trópicos.

Pero montar y mantener estaciones no es asunto sencillo. Las que se lograron deben mucho a las facilidades derivadas de la condición de colonia británica de Jamaica y Guyana y neocolonial de Cuba y Panamá. La mayoría operaron en terrenos que eran o habían sido plantaciones, aquellas empresas que aceleraron la tala de los bosques que los biólogos tanto admiraban. Tal conexión permitía que las estaciones lograran un "delicado balance entre la naturaleza primitiva y las comodidades de la

\footnotetext{
${ }^{3}$ Regina Horta Duarte, A biologia militante: o Museu Nacional, especialização científica, divulgação do conhecimento e práticas políticas no Brasil, 1926-1945 (Belo Horizonte: Ed. UFMG, 2010), una version corregida fue publicada en inglés como Activist Biology: The National Museum, Politics and Nation Building in Brazil (Tucson: The University of Arizona Press, 2016).
} 
civilización" (80). Debido a que surgió en el seno la mayor plantación azucarera de Cuba, la estación de Soledad era fundamentalmente agronómica y servía como base para estudios ecológicos y para la realización de cursos de campo (de la Universidad de Harvard). Soledad fue víctima de la revolución cubana, lo que enfatiza una vez más la estrecha relación entre prácticas científicas y relaciones políticas.

La reina de las estaciones fue la isla de Barro Colorado, la punta de un cerro que sobrevivió a las inundaciones con las que se creó el canal de Panamá. Inaugurada en 1923, Barro Colorado representó un modelo novedoso de estación. Además de ser un centro de investigaciones, esta isla fue también un espacio de conservación, favorecido por el aislamiento que implicaban las aguas de lago Gatún. Allí los investigadores reprodujeron el ambiente machista propio de su quehacer, al no permitir que las investigadoras pernoctaran en la isla, y racista, propio de su país, al mantener espacios y comida diferentes para científicos y trabajadores locales.

Estos espacios de investigación en el trópico, utilizados casi exclusivamente por biólogos estadounidenses, lograron legitimarse en parte por el papel que la naturaleza tropical adquirió en las discusiones sobre ecología. A mediados de siglo tomó vuelo el interés por descifrar los factores que explican la diversidad, utilizando para ello modelos matemáticos, tal como sucedía en otras disciplinas que querían asegurar su solidez y prestigio de la mano de la abstracción y la generalización. Las formas y colores de aves y plantas fueron traducidos a números y cálculos estadísticos, para la tranquilidad de muchos y la repulsión de otros. Raby explica con gran claridad estos y otros debates científicos que animaban a los protagonistas de esta historia.

Las estaciones científicas permitieron dimensionar la magnitud de la diversidad. A pesar de ser una isla relativamente pequeña, tomó medio siglo tener una idea más o menos completa de la vegetación de Barro Colorado. Además de casi ilimitada, la naturaleza tropical resultó ser mucho más dinámica de lo que se pensaba. Las estaciones también sirvieron para evidenciar la pérdida paulatina de esa abundancia. "La selva", en Costa Rica, que empezó como la finca de Leslie Holdridge pero pronto se convirtió en centro de estudios, fue quedando como un remanente del 
bosque en medio del pasto y los cultivos. De esa y otras experiencias fue tomando forma la convicción de que el ritmo de destrucción era muy rápido, la pérdida incalculable y la necesidad de desarrollo para evitar la catástrofe evidente. Son justamente esas las ideas sobre las que descansa la noción de biodiversidad popularizada a partir de 1986.

Aunque en múltiples ocasiones Raby menciona las relaciones de los investigadores estadounidenses con los latinoamericanos-como el caso de Thomas Barbour y el cubano Carlos de la Torre y Huerta-la historia que relata es, como lo sugiere el título, fundamentalmente estadounidense (o American, como dirían sin ningún atisbo de ironía Barbour y sus pares). La ciencia que retrata este libro está poco conectada con las instituciones locales, a diferencia, por ejemplo, de los esfuerzos de clasificación taxonómica que explora Camilo Quintero en Birds of Empire, Birds of Nation. ${ }^{4}$ Hay que continuar develando y analizando los esfuerzos latinoamericanos por dar sentido a la naturaleza tropical en diálogo con el excelente libro del que es objeto esta reseña. Ojalá contemos pronto con su traducción al español.

\section{REFERENCIAS}

Camilo Quintero, Birds of Empire, Birds of Nation: A Place for Science and Nature in U.S.-Colombia Relations (Bogotá: Ediciones Uniandes, 2012).

Megan Raby, American Tropics: The Caribbean Roots of Biodiversity Science (Chapel Hill, The University of North Carolina Press, 2017).

Regina Horta Duarte, Activist Biology: The National Museum, Politics and Nation Building in Brazil (Tucson: The University of Arizona Press, 2016).

Regina Horta Duarte, A Biologia militante: o Museu Nacional, especialização científica, divulgação do conhecimento e práticas políticas no Brasil, 1926-1945 (Belo Horizonte: Ed. UFMG, 2010).

\footnotetext{
${ }^{4}$ Camilo Quintero, Birds of Empire, Birds of Nation: A Place for Science and Nature in U.S.-Colombia Relations (Bogotá: Ediciones Uniandes, 2012).
} 
Reinaldo Funes Monzote, De bosque a sabana: azúcar, deforestación y medio ambiente en Cuba, 1942-1926 (México: Siglo Veintinuo Editores, Estado Libre y Soberano de Quintana Roo, 2000).

Reinaldo Funes Monzote, From Rainforest to Cane Field in Cuba: An Environmental History since 1492 (Chapel Hill: University of North Carolina Press, 2008).

Warren Dean, With Broadax and Firebrand: The Destruction of Brazil's Atlantic Forest (Berkeley: University of California Press, 1995). 